\title{
Incoherent pion photoproduction on the deuteron with polarization observables I. Formal expressions
}

\author{
H. Arenhövel and A. Fix \\ Institut für Kernphysik, Johannes Gutenberg-Universität Mainz, D-55099 Mainz, Germany
}

(Dated: April 6, 2021)

\begin{abstract}
Formal expressions are developed for the general five-fold differential cross section of incoherent $\pi$-photoproduction on the deuteron including beam and target polarization. The polarization observables of the cross section are described by various beam, target and beam-target asymmetries for polarized photons and/or polarized deuterons. They are given as bilinear hermitean forms in the reaction matrix elements divided by the unpolarized cross section. In addition, the corresponding observables for the semi-exclusive reaction $\vec{d}(\vec{\gamma}, \pi) N N$ are also given.
\end{abstract}

PACS numbers: 13.60.Le, 21.45.+v, 24.70.+s, 25.20.Lj

\section{INTRODUCTION}

Photoproduction of pions on light nuclei is an important topic in medium energy nuclear physics. It is motivated by different and complementary aspects. On the one hand one wants to study the elementary reaction on the neutron to which otherwise one has no access. On the other hand one is interested in the influence of a nuclear environment on the elementary production amplitude, and last but not least, one hopes to obtain information on nuclear structure.

Besides the study of unpolarized total and differential cross sections, polarization observables provide very often further insight into details of the underlying reaction mechanisms and possible structure effects. In this case, such observables will serve as additional critical tests or check points for theoretical models. The considerable progress in experimental techniques for studying polarization phenomena has brought into focus also the question, what role polarization effects play in pion photoproduction on nuclei. Of particular interest is photoproduction of pions on the deuteron in view of its simple structure. Indeed, it has been studied quite extensively over the past 50 years (see [1] and references therein). While in earlier work mainly total and semi-exclusive differential cross sections of incoherent pion production have been studied, polarization observables were considered more recently, both in experiment [2, 3] as well as in theory. For example, the spin asymmetry of the total cross section with respect to circular photon polarization, which determines the Gerasimov-Drell-Hearn sum rule, was investigated theoretically in [4, 5, 6$]$ and target asymmetries were considered in [7].

Subsequently, various polarization asymmetries of the semi-exclusive differential cross section $\vec{d}(\vec{\gamma}, \pi) N N$ were studied theoretically in a series of papers 8, 9, 10, 11, 12. Unfortunately, many of the results presented there are based on incorrect expressions for polarization observables, because the formal expressions for them were taken in analogy from the corresponding expressions of deuteron photodisintegration [13. This is in principle possible, since the spin degrees of freedom are the same in both reactions, provided one takes care to check where certain symmetry properties of the reaction amplitude have been used in the derivation of the polarization observables in photodisintegration, because they are not identical in both reactions. This caveat refers in particular to those observables which are related to linearly polarized photons. It appears that this fact was not taken into account so that the results in [9, 10] for them cannot be trusted. But also the results for circularly polarized photons are incorrect, namely the claim in 8], that all of them vanish identically, is wrong. Moreover, this statement is in contradiction to [10], where a non-vanishing differential spin asymmetry for circularly polarized photons is reported, because this asymmetry is proportional to the beam-target asymmetry $T_{10}^{c}$ for circularly polarized photons and a vector polarized deuteron, which means that the latter does not vanish. Thus, it is obvious that the importance of polarization effects requires a more careful and thorough treatment as done in $8,0,0,10,11,12$.

With the present work we want to provide a solid basis for the formal expressions of the various polarization observables which determine the differential cross section for incoherent pion production on the deuteron with polarized photons and/or polarized deuterons by deriving the general form of the differential cross section including all possible polarization asymmetries. It complements the work of Blaazer et al. 14], who have formally derived all possible polarization observables for coherent pion photoproduction on the deuteron. 


\section{KINEMATICS}

As a starting point, we will first consider the kinematics of the photoproduction reaction

$$
\gamma\left(k, \vec{\varepsilon}_{\mu}\right)+d\left(p_{d}\right) \rightarrow \pi(q)+N_{1}\left(p_{1}\right)+N_{2}\left(p_{2}\right),
$$

where we have defined the notation of the four-momenta of the participating particles. The circular polarization vector of the photon is denoted by $\vec{\varepsilon}_{\mu}$ with $\mu= \pm 1$. The following formal developments will not depend on the reference frame, laboratory or center-of-momentum (c.m.) frame. However, in view of our explicit application [15] in which the reaction is evaluated in the laboratory frame, we will refer sometimes to this frame for definiteness. We choose as independent variables for the description of the final state the outgoing pion momentum $\vec{q}=\left(q, \theta_{q}, \phi_{q}\right)$ and the spherical angles $\Omega_{p}=\left(\theta_{p}, \phi_{p}\right)$ of the relative momentum $\vec{p}=\left(\vec{p}_{1}-\vec{p}_{2}\right) / 2=\left(p, \Omega_{p}\right)$ of the two outgoing nucleons. Together with the incoming photon energy $\omega=k_{0}$, the momenta of the outgoing nucleons are fixed, i.e. $\vec{p}_{1 / 2}=\left(\vec{k}+\vec{p}_{d}-\vec{q}\right) / 2 \pm \vec{p}$. The coordinate system is chosen to have a right-handed orientation with $z$-axis along the photon momentum $\vec{k}$. We distinguish in general three planes: (i) the photon plane spanned by the photon momentum and the direction of maximal linear photon polarization, which defines the direction of the $x$-axis, (ii) the pion plane, spanned by the photon and pion momenta, which intersects the photon plane along the $z$-axis with an angle $\phi_{q}$, and (iii) the nucleon plane spanned by the momenta of the two outgoing nucleons intersecting the pion plane along the total momentum of the two nucleons. This is illustrated in Fig. प for the laboratory frame. In case that the linear photon polarization vanishes, one can choose $\phi_{q}=0$ and then photon and pion planes coincide.

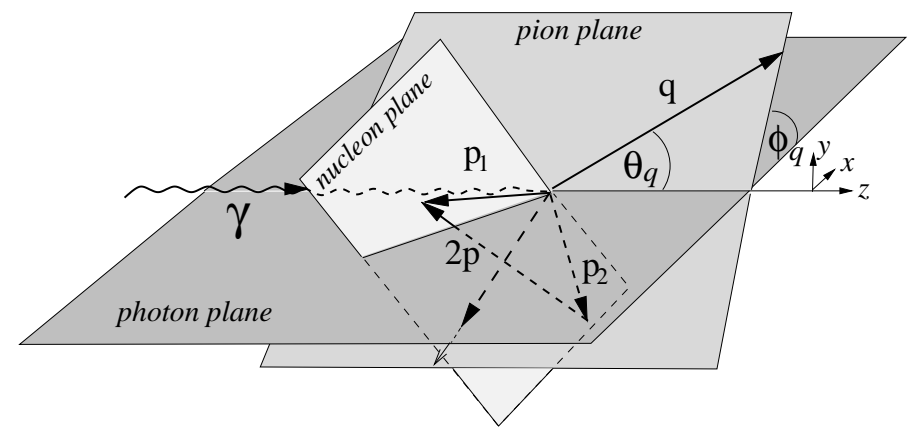

FIG. 1: Kinematics of pion photoproduction on the deuteron in the laboratory system.

\section{THE T-MATRIX}

All observables are determined by the $T$-matrix elements of the electromagnetic pion production current $\vec{J}_{\gamma \pi}$ between the initial deuteron and the final $\pi N N$ states. In a general frame, it is given by

$$
T_{s m_{s}, \mu m_{d}}=-{ }^{(-)}\left\langle\vec{p}_{1} \vec{p}_{2} s m_{s}, \vec{q}\left|\vec{\varepsilon}_{\mu} \cdot \vec{J}_{\gamma \pi}(0)\right| \vec{p}_{d} 1 m_{d}\right\rangle,
$$

where $s$ and $m_{s}$ denote the total spin and its projection on the relative momentum $\vec{p}$ of the outgoing two nucleons, and $m_{d}$ correspondingly the deuteron spin projection on the $z$-axis as quantization axis. Furthermore, transverse gauge has been chosen. The knowledge of the specific form of $\vec{J}_{\gamma \pi}$ is not needed for the following formal considerations.

The general form of the $T$-matrix after separation of the overall c.m.-motion is given by

$$
\begin{aligned}
T_{s m_{s} \mu m_{d}}\left(q, \Omega_{q}, \Omega_{p}\right) & ={ }^{(-)}\left\langle\vec{p} s m_{s}, \vec{q}\left|J_{\gamma \pi, \mu}(\vec{k})\right| 1 m_{d}\right\rangle \\
& =\sqrt{2 \pi} \sum_{L} i^{L} \hat{L}^{(-)}\left\langle\vec{p} s m_{s}, \vec{q}\left|\mathcal{O}_{\mu}^{\mu L}\right| 1 m_{d}\right\rangle
\end{aligned}
$$

with $\mu= \pm 1$ and transverse electric and magnetic multipoles

$$
\mathcal{O}_{M}^{\mu L}=E_{M}^{L}+\mu M_{M}^{L} .
$$


Furthermore, we use throughout the notation $\hat{L}=\sqrt{2 L+1}$. It is convenient to introduce a partial wave decomposition of the final states by

$$
\begin{aligned}
{ }^{(-)}\left\langle\vec{p} s m_{s}\right| & =\frac{1}{\sqrt{4 \pi}} \sum_{l_{p} j_{p} m_{p}} \hat{l}_{p}\left(l_{p} 0 s m_{s} \mid j_{p} m_{s}\right) D_{m_{s}, m_{p}}^{j_{p}}\left(\phi_{p},-\theta_{p},-\phi_{p}\right)^{(-)}\left\langle p\left(l_{p} s\right) j_{p} m_{p}\right| \\
(-)\langle\vec{q}| & =\frac{1}{\sqrt{4 \pi}} \sum_{l_{q} m_{q}} \hat{l}_{q} D_{0, m_{q}}^{l_{q}}\left(\phi_{q},-\theta_{q},-\phi_{q}\right)^{(-)}\left\langle q l_{q} m_{q}\right|
\end{aligned}
$$

where $m_{p}$ and $m_{q}$ like $m_{d}$ refer to the photon momentum $\vec{k}$ as quantization axis. Here, the rotation matrices $D_{m^{\prime} m}^{j}$ are taken in the convention of Rose [16]. Using the multipole decomposition and applying the Wigner-Eckart theorem yields

$$
\begin{gathered}
{ }^{(-)}\left\langle p\left(l_{p} s\right) j_{p} m_{p}, q l_{q} m_{q}\left|\mathcal{O}_{M}^{\mu L}\right| 1 m_{d}\right\rangle=\sum_{J M_{J}}(-)^{j_{p}-l_{q}+J} \hat{J}\left(\begin{array}{ccc}
j_{p} & l_{q} & J \\
m_{p} & m_{q} & -M_{J}
\end{array}\right)\left(\begin{array}{ccc}
J & L & 1 \\
-M_{J} & M & m_{d}
\end{array}\right) \\
\left\langle p q\left(\left(l_{p} s\right) j_{p} l_{q}\right) J \| \mathcal{O}^{\mu L}|| 1\right\rangle,
\end{gathered}
$$

with the selection rule $m_{p}+m_{q}=M_{J}=M+m_{d}$. Rewriting the angular dependence

$$
D_{m_{s}, m_{p}}^{j_{p}}\left(\phi_{p},-\theta_{p},-\phi_{p}\right) D_{0, m_{q}}^{l_{q}}\left(0,-\theta_{q},-\phi_{q}\right)=d_{m_{s}, m_{p}}^{j_{p}}\left(-\theta_{p}\right) d_{0, m_{q}}^{l_{q}}\left(-\theta_{q}\right) e^{i\left(\left(m_{p}-m_{s}\right) \phi_{p}+m_{q} \phi_{q}\right)},
$$

and rearranging, using the foregoing selection rule for $M=\mu$,

$$
\left(m_{p}-m_{s}\right) \phi_{p}+m_{q} \phi_{q}=\left(m_{p}-m_{s}\right) \phi_{p q}+\left(\mu+m_{d}-m_{s}\right) \phi_{q}
$$

with $\phi_{p q}=\phi_{p}-\phi_{q}$, one finds that the $T$-matrix can be written as

$$
T_{s m_{s} \mu m_{d}}\left(\Omega_{p}, \Omega_{q}\right)=e^{i\left(\mu+m_{d}-m_{s}\right) \phi_{q}} t_{s m_{s} \mu m_{d}}\left(\theta_{p}, \theta_{q}, \phi_{p q}\right),
$$

where the small $t$-matrix depends only on $\theta_{p}, \theta_{q}$, and the relative azimuthal angle $\phi_{p q}$. Explicitly one has

$$
\begin{aligned}
t_{s m_{s} \mu m_{d}}\left(\theta_{p}, \theta_{q}, \phi_{p q}\right)= & \frac{1}{2 \sqrt{2 \pi}} \sum_{L l_{p} j_{p} m_{p} l_{q} m_{q} J J M_{J}} i^{L} \hat{L} \hat{J} \hat{l}_{q} \hat{l}_{p} \hat{j}_{p}(-)^{J+l_{p}+j_{p}-s+m_{s}-l_{q}} \\
& \left(\begin{array}{ccc}
l_{p} & s & j_{p} \\
0 & m_{s} & -m_{s}
\end{array}\right)\left(\begin{array}{ccc}
j_{p} & l_{q} & J \\
m_{p} & m_{q} & -M_{J}
\end{array}\right)\left(\begin{array}{ccc}
J & L & 1 \\
-M_{J} & \mu & m_{d}
\end{array}\right) \\
& \left\langle p q\left(\left(l_{p} s\right) j_{p} l_{q}\right) J\left\|\mathcal{O}^{\mu L}\right\| 1\right\rangle d_{m_{s}, m_{p}}^{j_{p}}\left(-\theta_{p}\right) d_{0, m_{q}}^{l_{q}}\left(-\theta_{q}\right) e^{i\left(m_{p}-m_{s}\right) \phi_{p q}} .
\end{aligned}
$$

Using this explicit form for the small $t$-matrix, it is quite straightforward to show that, if parity is conserved, the following symmetry relation holds for the inverted spin projections

$$
t_{s-m_{s}-\mu-m_{d}}\left(\theta_{p}, \theta_{q}, \phi_{p q}\right)=(-)^{s+m_{s}+\mu+m_{d}} t_{s m_{s} \mu m_{d}}\left(\theta_{p}, \theta_{q},-\phi_{p q}\right) .
$$

In the derivation of this relation one has made use of the parity selection rules for the multipole transitions to a final partial wave $\left|p q\left(\left(l_{p} s\right) j_{p} l_{q}\right) J\right\rangle$ with parity $\pi_{J\left(l_{p}, l_{q}\right)}=(-)^{l_{p}+l_{q}+1}$

$$
\left\{\begin{array}{lll}
E^{L} & \pi_{d} \pi_{J\left(l_{p}, l_{q}\right)}(-)^{L}=1 & \rightarrow(-)^{l_{p}+l_{q}+L}=-1 \\
M^{L} & \pi_{d} \pi_{J\left(l_{p}, l_{q}\right)}(-)^{L}=-1 & \rightarrow(-)^{l_{p}+l_{q}+L}=1
\end{array}\right\} .
$$

Therefore, invariance under a parity transformation results in the following property of the reduced matrix element

$$
(-)^{l_{p}+l_{q}+L}\left\langle p q\left(\left(l_{p} s\right) j_{p} l_{q}\right) J\left\|\mathcal{O}^{-\mu L}\right\| 1\right\rangle=-\left\langle p q\left(\left(l_{p} s\right) j_{p} l_{q}\right) J\left\|\mathcal{O}^{\mu L}\right\| 1\right\rangle .
$$

The symmetry property (12) leads to a corresponding relation for the $T$-matrix

$$
T_{s-m_{s}-\mu-m_{d}}\left(\theta_{p}, \phi_{p}, \theta_{q}, \phi_{q}\right)=(-)^{s+m_{s}+\mu+m_{d}} T_{s m_{s} \mu m_{d}}\left(\theta_{p},-\phi_{p}, \theta_{q},-\phi_{q}\right) .
$$

For an uncoupled spin representation, one finds accordingly, using the transformation

$$
T_{m_{1} m_{2} \mu m_{d}}\left(\theta_{p}, \phi_{p}, \theta_{q}, \phi_{q}\right)=\sum_{s m_{s}}\left(\frac{1}{2} m_{1} \frac{1}{2} m_{2} \mid s m_{s}\right) T_{s m_{s} \mu m_{d}}\left(\theta_{p}, \phi_{p}, \theta_{q}, \phi_{q}\right),
$$

where $m_{j}$ denotes the spin projection of the "jth" nucleon on the quantization axis, as symmetry relation

$$
T_{-m_{1}-m_{2}-\mu-m_{d}}\left(\theta_{p}, \phi_{p}, \theta_{q}, \phi_{q}\right)=(-)^{1+m_{1}+m_{2}+\mu+m_{d}} T_{m_{1} m_{2} \mu m_{d}}\left(\theta_{p},-\phi_{p}, \theta_{q},-\phi_{q}\right) .
$$

The small $t$-matrix elements are the basic quantities which determine differential cross section and asymmetries. The latter are given as ratios of bilinear hermitean forms in terms of the $t$-matrix elements (see (37) and (38) below). 


\section{THE DIFFERENTIAL CROSS SECTION INCLUDING POLARIZATION ASYMMETRIES}

The usual starting point is the general expression for the differential cross section

$$
\frac{d^{5} \sigma}{d q d \Omega_{q} d \Omega_{p}}=c\left(\omega, q, \Omega_{q}, \Omega_{p}\right) \operatorname{tr}\left(T^{\dagger} T \rho_{i}\right),
$$

where $T$ denotes the reaction matrix, $\rho_{i}$ the density matrix for the spin degrees of the initial system. The trace refers to all initial and final state spin degrees of freedom comprising incoming photon, target deuteron, and final nucleons. Furthermore, $c\left(\omega, q, \Omega_{q}, \Omega_{p}\right)$ denotes a kinematic factor which comprises the final state phase space and the incoming flux. In an arbitrary frame one has

$$
c\left(\omega, q, \Omega_{q}, \Omega_{p}\right)=\frac{1}{(2 \pi)^{5}} \frac{E_{d}}{E_{d}+p_{d}} \frac{m_{N}^{2}}{4 \omega \omega_{\pi}} \frac{p^{*} q^{2}}{W_{N N}}
$$

with

$$
p^{*}=\frac{1}{2} \sqrt{W_{N N}^{2}-4 m_{N}^{2}},
$$

as the relative momentum of the final two nucleons in their c.m. sytem, and

$$
\omega=k_{0}, \quad E_{d}=\sqrt{p_{d}^{2}+m_{d}^{2}}, \quad \omega_{\pi}=\sqrt{q^{2}+m_{\pi}^{2}}, \quad W_{N N}^{2}=\left(\omega+E_{d}-\omega_{\pi}\right)^{2}-\left(\vec{k}+\vec{p}_{d}-\vec{q}\right)^{2} .
$$

The density matrix $\rho_{i}$ in (18) is a direct product of the density matrices $\rho^{\gamma}$ of the photon and $\rho^{d}$ of the deuteron

$$
\rho_{i}=\rho^{\gamma} \otimes \rho^{d}
$$

The photon density matrix has the form

$$
\rho_{\mu \mu^{\prime}}^{\gamma}=\frac{1}{2}\left(\delta_{\mu \mu^{\prime}}+\vec{P}^{\gamma} \cdot \vec{\sigma}_{\mu \mu^{\prime}}\right)
$$

with respect to circular polarization $\mu= \pm 1$. Here, $\left|\vec{P}^{\gamma}\right|$ describes the total degree of polarization, $P_{z}^{\gamma}=P_{c}^{\gamma}$ the degree of circular polarization, and $P_{l}^{\gamma}=\sqrt{\left(P_{x}^{\gamma}\right)^{2}+\left(P_{y}^{\gamma}\right)^{2}}$ the degree of linear polarization. By a proper rotation around the photon momentum, one can choose the $x$-axis in the direction of maximum linear polarization, i.e., $P_{x}^{\gamma}=-P_{l}^{\gamma}$ and $P_{y}^{\gamma}=0$. Then one has explicitly

$$
\rho_{\mu \mu^{\prime}}^{\gamma}=\left(1+\mu P_{c}^{\gamma}\right) \delta_{\mu \mu^{\prime}}-P_{l}^{\gamma} \delta_{\mu,-\mu^{\prime}} e^{2 i \mu \phi_{q}}
$$

Furthermore, the deuteron density matrix $\rho^{d}$ can be expressed in terms of irreducible spin operators $\tau^{[I]}$ with respect to the deuteron spin space

$$
\rho_{m_{d} m_{d^{\prime}}}^{d}=\frac{1}{3} \sum_{I M}(-)^{M} \hat{I}\left\langle 1 m_{d}\left|\tau_{M}^{[I]}\right| 1 m_{d}^{\prime}\right\rangle P_{I-M}^{d},
$$

where $P_{00}^{d}=1$, and $P_{1 M}^{d}$ and $P_{2 M}^{d}$ describe vector and tensor polarization components of the deuteron, respectively. The spin operators are defined by their reduced matrix elements

$$
\left\langle 1|| \tau^{[I]} \| 1\right\rangle=\sqrt{3} \hat{I} \quad \text { for } \quad I=0,1,2 .
$$

From now on we will assume that the deuteron density matrix is diagonal with respect to an orientation axis $\vec{d}$ having spherical angles $\left(\theta_{d}, \phi_{d}\right)$ with respect to the coordinate system associated with the photon plane in the lab frame. Then one has with respect to $\vec{d}$ as quantization axis

$$
\rho_{m m^{\prime}}^{d}=p_{m} \delta_{m m^{\prime}}
$$

where $p_{m}$ denotes the probability for finding a deuteron spin projection $m$ on the orientation axis. With respect to this axis one finds from (25) $P_{I M}^{d}(\vec{d})=P_{I}^{d} \delta_{M, 0}$, where the orientation parameters $P_{I}^{d}$ are related to the probabilities $\left\{p_{m}\right\}$ by

$$
\begin{aligned}
P_{I}^{d} & =\sqrt{3} \hat{I} \sum_{m}(-)^{1-m}\left(\begin{array}{ccc}
1 & 1 & I \\
m & -m & 0
\end{array}\right) p_{m} \\
& =\delta_{I 0}+\sqrt{\frac{3}{2}}\left(p_{1}-p_{-1}\right) \delta_{I 1}+\frac{1}{\sqrt{2}}\left(1-3 p_{0}\right) \delta_{I 2} .
\end{aligned}
$$


The polarization components in the chosen lab frame are obtained from the $P_{I}^{d}$ by a rotation, transforming the quantization axis along the orientation axis into the direction of the photon momentum, i.e.

$$
P_{I M}^{d}(\vec{z})=P_{I}^{d} e^{i M \phi_{d}} d_{M 0}^{I}\left(\theta_{d}\right)
$$

where $d_{m m^{\prime}}^{j}$ denotes a small rotation matrix [16]. Thus the deuteron density matrix becomes finally

$$
\rho_{m_{d} m_{d^{\prime}}}^{d}=\frac{1}{\sqrt{3}}(-)^{1-m_{d}} \sum_{I M} \hat{I}\left(\begin{array}{ccc}
1 & 1 & I \\
m_{d}^{\prime} & -m_{d} & M
\end{array}\right) P_{I}^{d} e^{-i M \phi_{d}} d_{M 0}^{I}\left(\theta_{d}\right) .
$$

This means, the deuteron target is characterized by four parameters, namely the vector and tensor polarization parameters $P_{1}^{d}$ and $P_{2}^{d}$, respectively, and by the orientation angles $\theta_{d}$ and $\phi_{d}$. If one chooses the c.m. frame as reference frame, one should note that the deuteron density matrix undergoes no change in the transformation from the lab to the c.m. system, since the boost to the c.m. system is collinear with the deuteron quantization axis [17].

The evaluation of the general expression of the differential cross section in (18) can be done analogously to deuteron photodisintegration as described in detail in 13]. In fact, one can follow the same steps except for the use of the symmetry relation of Eq. (2) in 13] which is different in case of pion production (see (12)) because of the additional pion degree of freedom in the final state, in particular its pseudovector character. In terms of the small $t$-matrices as defined in (10), one finds, inserting the density matrices of photon and deuteron for the general five-fold differential cross section,

$$
\frac{d^{5} \sigma}{d q d \Omega_{q} d \Omega_{p}}=\frac{1}{2} \sum_{\mu^{\prime} \mu I M} P_{I}^{d} e^{i M\left(\phi_{q}-\phi_{d}\right)} d_{M 0}^{I}\left(\theta_{d}\right) u_{I M}^{\mu^{\prime} \mu}\left[\left(1+\mu P_{c}^{\gamma}\right) \delta_{\mu \mu^{\prime}}-P_{l}^{\gamma} \delta_{\mu,-\mu^{\prime}} e^{2 i \mu \phi_{q}}\right]
$$

where we have introduced the quantities

$$
\begin{aligned}
u_{I M}^{\mu^{\prime} \mu}\left(q, \theta_{q}, \theta_{p}, \phi_{p q}\right)= & c\left(\omega, q, \Omega_{q}, \Omega_{p}\right) \frac{\hat{I}}{\sqrt{3}} \sum_{m_{d} m_{d}^{\prime}}(-)^{1-m_{d}}\left(\begin{array}{ccc}
1 & 1 & I \\
m_{d}^{\prime} & -m_{d} & M
\end{array}\right) \\
& \sum_{s m_{s}} t_{s m_{s} \mu^{\prime} m_{d}^{\prime}}^{*}\left(q, \theta_{q}, \theta_{p}, \phi_{p q}\right) t_{s m_{s} \mu m_{d}}\left(q, \theta_{q}, \theta_{p}, \phi_{p q}\right) .
\end{aligned}
$$

It is straighforward to prove that they behave under complex conjugation as

$$
u_{I M}^{\mu^{\prime} \mu}\left(q, \theta_{q}, \theta_{p}, \phi_{p q}\right)^{*}=(-)^{M} u_{I-M}^{\mu \mu^{\prime}}\left(q, \theta_{q}, \theta_{p}, \phi_{p q}\right) .
$$

Furthermore, with the help of the symmetry in (12) one finds

$$
u_{I M}^{-\mu^{\prime}-\mu}\left(q, \theta_{q}, \theta_{p}, \phi_{p q}\right)=(-)^{I+M+\mu^{\prime}+\mu} u_{I-M}^{\mu^{\prime} \mu}\left(q, \theta_{q}, \theta_{p},-\phi_{p q}\right),
$$

which yields in combination with (33)

$$
u_{I M}^{-\mu^{\prime}-\mu}\left(q, \theta_{q}, \theta_{p}, \phi_{p q}\right)=(-)^{I+\mu^{\prime}+\mu} u_{I M}^{\mu \mu^{\prime}}\left(q, \theta_{q}, \theta_{p},-\phi_{p q}\right)^{*}
$$

This relation is quite useful for a further simplification of the semi-exclusive differential cross section later on.

Separating the polarization parameters of photon $\left(P_{l}^{\gamma}\right.$ and $\left.P_{c}^{\gamma}\right)$ and deuteron $\left(P_{I}^{d}\right)$, it is then straightforward to show that the differential cross section can be brought into the form

$$
\begin{aligned}
\frac{d^{5} \sigma}{d q d \Omega_{q} d \Omega_{p}}= & \frac{1}{2} \sum_{I} P_{I}^{d} \sum_{M=-I}^{I} e^{i M \phi_{q d}} d_{M 0}^{I}\left(\theta_{d}\right)\left[v_{I M}^{1}+v_{I M}^{-1}\right. \\
& \left.+P_{c}^{\gamma}\left(v_{I M}^{1}-v_{I M}^{-1}\right)+P_{l}^{\gamma}\left(w_{I M}^{1} e^{-2 i \phi_{q}}+w_{I M}^{-1} e^{2 i \phi_{q}}\right)\right]
\end{aligned}
$$

with $\phi_{q d}=\phi_{q}-\phi_{d}$, where we have introduced for convenience the quantities

$$
\begin{aligned}
v_{I M}^{\mu}\left(q, \theta_{q}, \theta_{p}, \phi_{p q}\right) & =u_{I M}^{\mu \mu}\left(q, \theta_{q}, \theta_{p}, \phi_{p q}\right) \\
w_{I M}^{\mu}\left(q, \theta_{q}, \theta_{p}, \phi_{p q}\right) & =-u_{I M}^{\mu-\mu}\left(q, \theta_{q}, \theta_{p}, \phi_{p q}\right) .
\end{aligned}
$$


According to (33) and (35), they have the following properties under complex conjugation

$$
\begin{aligned}
v / w_{I M}^{\mu}\left(q, \theta_{q}, \theta_{p}, \phi_{p q}\right)^{*} & =(-)^{M} v / w_{I-M}^{\mu}\left(q, \theta_{q}, \theta_{p}, \phi_{p q}\right), \\
v_{I M}^{\mu}\left(q, \theta_{q}, \theta_{p}, \phi_{p q}\right)^{*} & =(-)^{I} v_{I M}^{-\mu}\left(q, \theta_{q}, \theta_{p},-\phi_{p q}\right), \\
w_{I M}^{\mu}\left(q, \theta_{q}, \theta_{p}, \phi_{p q}\right)^{*} & =(-)^{I} w_{I M}^{\mu}\left(q, \theta_{q}, \theta_{p},-\phi_{p q}\right) .
\end{aligned}
$$

From Eq. (39) follows that $v_{I 0}^{\mu}$ and $w_{I 0}^{\mu}$ are real. The sum over $M$ in (36) can be rearranged with the help of the relation (39) and $d_{-M 0}^{I}\left(\theta_{d}\right)=(-)^{M} d_{M 0}^{I}\left(\theta_{d}\right)$

$$
\begin{aligned}
\sum_{M=-I}^{I} e^{i M \phi_{q d}} d_{M 0}^{I}\left(\theta_{d}\right)\left(v_{I M}^{1} \pm v_{I M}^{-1}\right) & =\sum_{M=0}^{I} \frac{d_{M 0}^{I}\left(\theta_{d}\right)}{1+\delta_{M 0}}\left(e^{i M \phi_{q d}}\left(v_{I M}^{1} \pm v_{I M}^{-1}\right)+e^{-i M \phi_{q d}}(-)^{M}\left(v_{I-M}^{1} \pm v_{I-M}^{-1}\right)\right) \\
& =\sum_{M=0}^{I} \frac{d_{M 0}^{I}\left(\theta_{d}\right)}{1+\delta_{M 0}}\left(e^{i M \phi_{q d}}\left(v_{I M}^{1} \pm v_{I M}^{-1}\right)+\text { c.c. }\right),
\end{aligned}
$$

and furthermore with $\psi_{M}=M \phi_{q d}-2 \phi_{q}$

$$
\begin{aligned}
\sum_{M=-I}^{I} e^{i M \phi_{q d}} d_{M 0}^{I}\left(\theta_{d}\right)\left(w_{I M}^{1} e^{-2 i \phi_{q}}+w_{I M}^{-1} e^{2 i \phi_{q}}\right) & =\sum_{M=-I}^{I} d_{M 0}^{I}\left(\theta_{d}\right)\left(e^{i \psi_{M}} w_{I M}^{1}+e^{-i \psi_{M}}(-)^{M} w_{I-M}^{-1}\right) \\
& =\sum_{M=-I}^{I} d_{M 0}^{I}\left(\theta_{d}\right)\left(e^{i \psi_{M}} w_{I M}^{1}+\text { c.c. }\right) .
\end{aligned}
$$

This then yields for the differential cross section

$$
\begin{aligned}
\frac{d^{5} \sigma}{d q d \Omega_{q} d \Omega_{p}}= & \sum_{I} P_{I}^{d}\left\{\sum_{M=0}^{I} \frac{1}{1+\delta_{M 0}} d_{M 0}^{I}\left(\theta_{d}\right) \Re e\left[e^{i M \phi_{q d}}\left(v_{I M}^{+}+P_{c}^{\gamma} v_{I M}^{-}\right)\right]\right. \\
& \left.+P_{l}^{\gamma} \sum_{M=-I}^{I} d_{M 0}^{I}\left(\theta_{d}\right) \Re e\left[e^{i \psi_{M}} w_{I M}^{1}\right]\right\}
\end{aligned}
$$

where we have defined

$$
v_{I M}^{ \pm}=v_{I M}^{1} \pm v_{I M}^{-1} .
$$

Now, introducing various beam, target and beam-target asymmetries by

$$
\begin{aligned}
\tau_{I M}^{0 / c}\left(q, \theta_{q}, \theta_{p}, \phi_{p q}\right) & =\frac{1}{1+\delta_{M 0}} \Re e v_{I M}^{ \pm}\left(q, \theta_{q}, \theta_{p}, \phi_{p q}\right), \quad M \geq 0, \\
\sigma_{I M}^{0 / c}\left(q, \theta_{q}, \theta_{p}, \phi_{p q}\right) & =-\Im m v_{I M}^{ \pm}\left(q, \theta_{q}, \theta_{p}, \phi_{p q}\right), \quad M>0, \\
\tau_{I M}^{l}\left(q, \theta_{q}, \theta_{p}, \phi_{p q}\right) & =\Re e w_{I M}^{1}\left(q, \theta_{q}, \theta_{p}, \phi_{p q}\right), \\
\sigma_{I M}^{l}\left(q, \theta_{q}, \theta_{p}, \phi_{p q}\right) & =-\Im m w_{I M}^{1}\left(q, \theta_{q}, \theta_{p}, \phi_{p q}\right), \quad M \neq 0,
\end{aligned}
$$

where we took into account that $v_{I 0}^{\mu}$ and $w_{I 0}^{\mu}$ are real, one obtains as final expression for the general five-fold differential cross section with beam and target polarization

$$
\begin{aligned}
\frac{d^{5} \sigma}{d q d \Omega_{q} d \Omega_{p}}= & \sum_{I} P_{I}^{d}\left\{\sum _ { M = 0 } ^ { I } d _ { M 0 } ^ { I } ( \theta _ { d } ) \left[\tau_{I M}^{0} \cos \left(M \phi_{q d}\right)+\sigma_{I M}^{0} \sin \left(M \phi_{q d}\right)\right.\right. \\
& \left.+P_{c}^{\gamma}\left(\tau_{I M}^{c} \cos \left(M \phi_{q d}\right)+\sigma_{I M}^{c} \sin \left(M \phi_{q d}\right)\right)\right] \\
& \left.+P_{l}^{\gamma} \sum_{M=-I}^{I} d_{M 0}^{I}\left(\theta_{d}\right)\left[\tau_{I M}^{l} \cos \psi_{M}+\sigma_{I M}^{l} \sin \psi_{M}\right]\right\} .
\end{aligned}
$$

This constitutes our central result. 
We will now turn to the semi-exclusive reaction $\vec{d}(\vec{\gamma}, \pi) N N$ where only the produced pion is detected, which means integration of the five-fold differential cross section $d^{5} \sigma / d q d \Omega_{q} d \Omega_{p}$ over $\Omega_{p}$. The resulting cross section will then be governed by the integrated asymmetries $\int d \Omega_{p} \tau_{I M}^{\alpha}$ and $\int d \Omega_{p} \sigma_{I M}^{\alpha}(\alpha \in\{0, c, l\})$, of which quite a few will vanish, either $\int d \Omega_{p} \tau_{I M}^{\alpha}$ or $\int d \Omega_{p} \sigma_{I M}^{\alpha}$. To show this, we first introduce the quantities

$$
\begin{aligned}
W_{I M}\left(q, \theta_{q}\right)= & \int d \Omega_{p} w_{I M}^{1}\left(q, \theta_{q}, \theta_{p}, \phi_{p q}\right) \\
= & -\frac{\hat{I}}{\sqrt{3}} \int d \Omega_{p} c\left(\omega, q, \Omega_{q}, \Omega_{p}\right) \sum_{m_{d} m_{d}^{\prime}}(-)^{1-m_{d}}\left(\begin{array}{ccc}
1 & 1 & I \\
m_{d}^{\prime} & -m_{d} & M
\end{array}\right) \\
& \sum_{s m_{s}} t_{s m_{s} 1 m_{d}^{\prime}}^{*}\left(q, \theta_{q}, \theta_{p}, \phi_{p q}\right) t_{s m_{s}-1 m_{d}}\left(q, \theta_{q}, \theta_{p}, \phi_{p q}\right), \\
V_{I M}^{ \pm}\left(q, \theta_{q}\right)= & V_{I M}^{1}\left(q, \theta_{q}\right) \pm V_{I M}^{-1}\left(q, \theta_{q}\right),
\end{aligned}
$$

with

$$
\begin{aligned}
V_{I M}^{\mu}\left(q, \theta_{q}\right)= & \int d \Omega_{p} v_{I M}^{\mu}\left(q, \theta_{q}, \theta_{p}, \phi_{p q}\right) \\
= & \frac{\hat{I}}{\sqrt{3}} \int d \Omega_{p} c\left(\omega, q, \Omega_{q}, \Omega_{p}\right) \sum_{m_{d} m_{d}^{\prime}}(-)^{1-m_{d}}\left(\begin{array}{ccc}
1 & 1 & I \\
m_{d}^{\prime} & -m_{d} & M
\end{array}\right) \\
& \sum_{s m_{s}} t_{s m_{s} \mu m_{d}^{\prime}}^{*}\left(q, \theta_{q}, \theta_{p}, \phi_{p q}\right) t_{s m_{s} \mu m_{d}}\left(q, \theta_{q}, \theta_{p}, \phi_{p q}\right) .
\end{aligned}
$$

Using now the property (40), one finds with the help of

$$
\int_{0}^{2 \pi} d \phi_{p} f\left(-\phi_{p q}\right)=\int_{0}^{2 \pi} d \phi_{p} f\left(\phi_{p q}\right)
$$

for a periodic function $f\left(\phi_{p q}+2 \pi\right)=f\left(\phi_{p q}\right)$, the relation

$$
V_{I M}^{-1}\left(q, \theta_{q}\right)=\int d \Omega_{p} v_{I M}^{-1}\left(q, \theta_{q}, \theta_{p}, \phi_{p q}\right)=(-)^{I} \int d \Omega_{p} v_{I M}^{1}\left(q, \theta_{q}, \theta_{p},-\phi_{p q}\right)^{*}=(-)^{I} V_{I M}^{1}\left(q, \theta_{q}\right)^{*},
$$

and thus

$$
V_{I M}^{ \pm}\left(q, \theta_{q}\right)=V_{I M}^{1}\left(q, \theta_{q}\right) \pm(-)^{I} V_{I M}^{1}\left(q, \theta_{q}\right)^{*} .
$$

Correspondingly, using (41) one obtains

$$
W_{I M}\left(q, \theta_{q}\right)^{*}=(-)^{I} \int d \Omega_{p} w_{I M}^{1}\left(q, \theta_{q}, \theta_{p},-\phi_{p q}\right)=(-)^{I} W_{I M}\left(q, \theta_{q}\right) .
$$

From the two foregoing equations we can conclude that $V_{I M}^{+}$and $W_{I M}$ are real for $I=0$ and 2 and imaginary for $I=1$, whereas $V_{I M}^{-}$is imaginary for $I=0$ and 2 and real for $I=1$. Therefore, according to (46) through (49) the following integrated asymmetries vanish

$$
\begin{gathered}
\int d \Omega_{p} \tau_{I M}^{\alpha}=0 \text { for }\left\{\begin{array}{ll}
\alpha \in\{0, l\}, & \text { and } I=1 \\
\alpha \in\{c\}, & \text { and } I=0,2
\end{array}\right\}, \\
\int d \Omega_{p} \sigma_{I M}^{\alpha}=0 \text { for }\left\{\begin{array}{ll}
\alpha \in\{0, l\}, & \text { and } I=0,2 \\
\alpha \in\{c\}, & \text { and } I=1
\end{array}\right\} .
\end{gathered}
$$

Instead of using these results for deriving from (50) the three-fold semi-exclusive differential cross section, we prefer to start from the expression in (44), and obtain

$$
\frac{d^{3} \sigma}{d q d \Omega_{q}}=\sum_{I} P_{I}^{d}\left\{\sum_{M=0}^{I} \frac{1}{1+\delta_{M 0}} d_{M 0}^{I}\left(\theta_{d}\right) \Re e\left[e^{i M \phi_{q d}}\left(V_{I M}^{+}+P_{c}^{\gamma} V_{I M}^{-}\right)\right]+P_{l}^{\gamma} \sum_{M=-I}^{I} d_{M 0}^{I}\left(\theta_{d}\right) \Re e\left[e^{i \psi_{M}} W_{I M}\right]\right\} .
$$


This expression can be simplified using the fact that $i^{\delta_{I 1}} W_{I M}, i^{\delta_{I 1}} V_{I M}^{+}$and $i^{1-\delta_{I 1}} V_{I M}^{-}$are real according to (56) and (57). The latter two quantities can be written as

$$
\begin{aligned}
i^{\delta_{I 1}} V_{I M}^{+} & =2 \Re e\left(i^{\delta_{I 1}} V_{I M}^{1}\right), \\
i^{1-\delta_{I 1}} V_{I M}^{-} & =2 \Re e\left(i^{1-\delta_{I 1}} V_{I M}^{1}\right)=-2 \Im m\left(i^{-\delta_{I 1}} V_{I M}^{1}\right) .
\end{aligned}
$$

Using now

$$
\begin{aligned}
\Re e\left[e^{i M \phi_{q d}} V_{I M}^{+}\right] & =\Re e\left[e^{i\left(M \phi_{q d}-\delta_{I 1} \pi / 2\right)} i^{\delta_{I 1}} V_{I M}^{+}\right]=2 \Re e\left(i^{\delta_{I 1}} V_{I M}^{1}\right) \cos \left[M \phi_{q d}-\delta_{I 1} \pi / 2\right], \\
\Re e\left[e^{i M \phi_{q d}} V_{I M}^{-}\right] & =\Re e\left[\frac{1}{i} e^{i\left(M \phi_{q d}+\delta_{I 1} \pi / 2\right)} i^{1-\delta_{I 1}} V_{I M}^{-}\right]=-2 \Im m\left(i^{-\delta_{I 1}} V_{I M}^{1}\right) \sin \left[M \phi_{q d}+\delta_{I 1} \pi / 2\right], \\
\Re e\left[e^{i \psi_{M}} W_{I M}\right] & =\Re e\left[e^{i\left(\psi_{M}-\delta_{I 1} \pi / 2\right)} i^{\delta_{I 1}} W_{I M}\right]=i^{\delta_{I 1}} W_{I M} \cos \left[\psi_{M}-\delta_{I 1} \pi / 2\right],
\end{aligned}
$$

we find as final form for the three-fold semi-exclusive differential cross section

$$
\begin{aligned}
\frac{d^{3} \sigma}{d q d \Omega_{q}}= & \frac{d^{3} \sigma_{0}}{d q d \Omega_{q}}\left[1+P_{l}^{\gamma}\left\{\widetilde{\Sigma}^{l} \cos 2 \phi_{q}+\sum_{I=1}^{2} P_{I}^{d} \sum_{M=-I}^{I} \widetilde{T}_{I M}^{l} \cos \left[\psi_{M}-\delta_{I 1} \pi / 2\right] d_{M 0}^{I}\left(\theta_{d}\right)\right\}\right. \\
& \left.+\sum_{I=1}^{2} P_{I}^{d} \sum_{M=0}^{I}\left(\widetilde{T}_{I M}^{0} \cos \left[M \phi_{q d}-\delta_{I 1} \pi / 2\right]+P_{c}^{\gamma} \widetilde{T}_{I M}^{c} \sin \left[M \phi_{q d}+\delta_{I 1} \pi / 2\right]\right) d_{M 0}^{I}\left(\theta_{d}\right)\right]
\end{aligned}
$$

Here the unpolarized cross section and the asymmetries are given by

$$
\begin{aligned}
\frac{d^{3} \sigma_{0}}{d q d \Omega_{q}} & =V_{00}^{1}\left(q, \theta_{q}\right), \\
\widetilde{\Sigma}^{l}\left(q, \theta_{q}\right) \frac{d^{3} \sigma_{0}}{d q d \Omega_{q}} & =W_{00}\left(q, \theta_{q}\right), \\
\widetilde{T}_{I M}^{0}\left(q, \theta_{q}\right) \frac{d^{3} \sigma_{0}}{d q d \Omega_{q}} & =\left(2-\delta_{M 0}\right) \Re e\left[i^{\delta_{I 1}} V_{I M}^{1}\left(q, \theta_{q}\right)\right], \quad \text { for } 0 \leq M \leq I, \\
\widetilde{T}_{I M}^{c}\left(q, \theta_{q}\right) \frac{d^{3} \sigma_{0}}{d q d \Omega_{q}} & =-\left(2-\delta_{M 0}\right) \Im m\left[i^{-\delta_{I 1}} V_{I M}^{1}\left(q, \theta_{q}\right)\right], \quad \text { for } 0 \leq M \leq I, \\
\widetilde{T}_{I M}^{l}\left(q, \theta_{q}\right) \frac{d^{3} \sigma_{0}}{d q d \Omega_{q}} & =i^{\delta_{I 1}} W_{I M}\left(q, \theta_{q}\right), \quad \text { for }-I \leq M \leq I .
\end{aligned}
$$

Because $V_{I 0}^{1}$ is real according to (39), the asymmetries $\widetilde{T}_{10}$ and $\widetilde{T}_{20}^{c}$ vanish identically. We would like to point out that in forward and backward pion emission, i.e. for $\theta_{q}=0$ and $\pi$, the following asymmetries have to vanish

$$
\widetilde{\Sigma}^{l}=0, \quad \widetilde{T}_{I M}^{0, c}=0 \text { for } M \neq 0, \quad \text { and } \quad T_{I M}^{l}=0 \text { for } M \neq 2,
$$

because in that case the differential cross section cannot depend on $\phi_{q}$, since at $\theta_{q}=0$ or $\pi$ the azimuthal angle $\phi_{q}$ is undefined or arbitrary. This feature can also be shown by straightforward evaluation of $V_{I M}^{\mu}$ and $W_{I M}$ using the explicit representation of the $t$-matrix in (11). One finds

$$
V_{I M}^{\mu}\left(q, \theta_{q}=0 / \pi, \theta_{p}, \phi_{p q}\right)=0 \quad \text { for } M \neq 0 \quad \text { and } \quad W_{I M}\left(q, \theta_{q}=0 / \pi, \theta_{p}, \phi_{p q}\right)=0 \quad \text { for } M \neq 2 .
$$

The authors of [12] were not aware of this general kinematic property because they evaluate the asymmetries numerically for $\theta_{q}=0$ and $\pi$ and find that the obtained values are of the order of $10^{-3}$. They conclude in the case of $T_{11}$ that it vanishes there but point out that $\Sigma^{l}$ does not vanish. For completeness and also in view of the numerous errors in $8,9,10,12$, we list in the appendix . the explicit expressions of the asymmetries in terms of the $t$-matrix elements.

In case that only the direction of the outgoing pion is measured and not its momentum, the corresponding differential cross section $d^{2} \sigma / d \Omega_{q}$ is given by an expression formally analogous to [66) where only the above asymmetries are integrated over the pion momentum, i.e., by the replacements

$$
\begin{aligned}
\frac{d^{3} \sigma_{0}}{d q d \Omega_{q}} & \rightarrow \frac{d^{2} \sigma_{0}}{d \Omega_{q}}=\int_{q_{\min }\left(\theta_{q}\right)}^{q_{\max }\left(\theta_{q}\right)} d q \frac{d^{3} \sigma_{0}}{d q d \Omega_{q}}, \\
\frac{d^{3} \sigma_{0}}{d q d \Omega_{q}} \widetilde{\Sigma}^{l}\left(q, \theta_{q}\right) & \rightarrow \frac{d^{2} \sigma_{0}}{d \Omega_{q}} \Sigma^{l}\left(\theta_{q}\right)=\int_{q_{\min }\left(\theta_{q}\right)}^{q_{\max }\left(\theta_{q}\right)} d q \frac{d^{3} \sigma_{0}}{d q d \Omega_{q}} \widetilde{\Sigma}^{l}\left(q, \theta_{q}\right), \\
\frac{d^{3} \sigma_{0}}{d q d \Omega_{q}} \widetilde{T}_{I M}^{\alpha}\left(q, \theta_{q}\right) & \rightarrow \frac{d^{2} \sigma_{0}}{d \Omega_{q}} T_{I M}^{\alpha}\left(\theta_{q}\right)=\int_{q_{\min }\left(\theta_{q}\right)}^{q_{\max }\left(\theta_{q}\right)} d q \frac{d^{3} \sigma_{0}}{d q d \Omega_{q}} \widetilde{T}_{I M}^{\alpha}\left(q, \theta_{q}\right), \quad \alpha \in\{0, l, c\} .
\end{aligned}
$$


The upper and lower integration limits are given by

$$
\begin{aligned}
& q_{\max }\left(\theta_{q}\right)=\frac{1}{2 b}\left(a \omega \cos \theta_{q}+E_{\gamma d} \sqrt{a^{2}-4 b m_{\pi}^{2}}\right), \\
& q_{\min }\left(\theta_{q}\right)=\max \left\{0, \frac{1}{2 b}\left(a \omega \cos \theta_{q}-E_{\gamma d} \sqrt{a^{2}-4 b m_{\pi}^{2}}\right)\right\},
\end{aligned}
$$

where

$$
\begin{aligned}
a & =W_{\gamma d}^{2}+m_{\pi}^{2}-4 m_{N}^{2}, \\
b & =W_{\gamma d}^{2}+\omega^{2} \sin ^{2} \theta_{q}, \\
W_{\gamma d}^{2} & =m_{d}\left(m_{d}+2 \omega\right), \\
E_{\gamma d} & =m_{d}+\omega .
\end{aligned}
$$

The general total cross section is obtained from (66) by integrating over $q$ and $\Omega_{q}$ resulting in

$$
\sigma\left(P_{l}^{\gamma}, P_{c}^{\gamma}, P_{1}^{d}, P_{2}^{d}\right)=\sigma_{0}\left[1+P_{2}^{d} \bar{T}_{20}^{0} \frac{1}{2}\left(3 \cos ^{2} \theta_{d}-1\right)+P_{c}^{\gamma} P_{1}^{d} \bar{T}_{10}^{c} \cos \theta_{d}+P_{l}^{\gamma} P_{2}^{d} \bar{T}_{22}^{l} \cos \left(2 \phi_{d}\right) \frac{\sqrt{6}}{4} \sin ^{2} \theta_{d}\right],
$$

where the unpolarized total cross section and the corresponding asymmetries are given by

$$
\begin{aligned}
\sigma_{0} & =\int d \Omega_{q} \int_{q_{\min }\left(\theta_{q}\right)}^{q_{\max }\left(\theta_{q}\right)} d q \frac{d^{3} \sigma_{0}}{d q d \Omega_{q}}, \\
\sigma_{0} \bar{T}_{I M}^{\alpha} & =\int d \Omega_{q} \int_{q_{\min }\left(\theta_{q}\right)}^{q_{\max }\left(\theta_{q}\right)} d q \frac{d^{3} \sigma_{0}}{d q d \Omega_{q}} \widetilde{T}_{I M}^{\alpha},
\end{aligned}
$$

with $\alpha \in\{0, l, c\}$.

Finally, we would like to point out that for coherent photoproduction of $\pi^{0}$ on the deuteron formally the same expression as in (66) holds with unpolarized differential cross section and asymmetries $\Sigma^{l}\left(\theta_{q}\right), T_{I M}\left(\theta_{q}\right)$, and $T_{I M}^{c / l}\left(\theta_{q}\right)$, which are defined in analogy to (67) through (71) with the replacements

$$
\begin{aligned}
V_{I M}^{1} & \rightarrow c\left(\omega, \Omega_{q}\right) \frac{\hat{I}}{\sqrt{3}} \sum_{m_{d} m_{d}^{\prime}}(-)^{1-m_{d}}\left(\begin{array}{ccc}
1 & 1 & I \\
m_{d}^{\prime} & -m_{d} & M
\end{array}\right) \sum_{m_{d}^{\prime \prime}} t_{m_{d}^{\prime \prime} 1 m_{d}^{\prime}}\left(\theta_{q}\right) t_{m_{d}^{\prime \prime} 1 m_{d}}\left(\theta_{q}\right), \\
W_{I M} & \rightarrow-c\left(\omega, \Omega_{q}\right) \frac{\hat{I}}{\sqrt{3}} \sum_{m_{d} m_{d}^{\prime}}(-)^{1-m_{d}}\left(\begin{array}{ccc}
1 & 1 & I \\
m_{d}^{\prime} & -m_{d} & M
\end{array}\right) \sum_{m_{d}^{\prime \prime}} t_{m_{d}^{\prime \prime} 1 m_{d}^{\prime}}^{*}\left(\theta_{q}\right) t_{m_{d}^{\prime \prime}-1 m_{d}}\left(\theta_{q}\right) .
\end{aligned}
$$

Here, $c\left(\omega, \Omega_{q}\right)$ denotes a kinematic factor. A complete listing of all polarization observables including recoil polarization of the final deuteron can be found in [14].

\section{CONCLUSIONS}

In this work we have derived formal expressions for the differential cross section of incoherent pion photoproduction on the deuteron including various polarization asymmetries with respect to polarized photons and deuterons. Obviously, these expressions are generally valid for pseudoscalar meson production. We did not consider polarization analysis of the final state, i.e. spin analysis of one or both outgoing nucleons. In this case one has to evaluate instead of (18)

$$
P_{\alpha}(j) \frac{d^{5} \sigma}{d q d \Omega_{q} d \Omega_{p}}=c\left(\omega, q, \Omega_{q}, \Omega_{p}\right) \operatorname{tr}\left(T^{\dagger} \sigma_{\alpha}(j) T \rho_{i}\right),
$$

for the polarization of the "jth" outgoing nucleon, or

$$
P_{\alpha_{1} \alpha_{2}} \frac{d^{5} \sigma}{d q d \Omega_{q} d \Omega_{p}}=c\left(\omega, q, \Omega_{q}, \Omega_{p}\right) \operatorname{tr}\left(T^{\dagger} \sigma_{\alpha_{1}}(1) \sigma_{\alpha_{2}}(2) T \rho_{i}\right),
$$

for the polarization of both outgoing nucleons. For the evaluation of these expressions one can proceed straightforwardly as has been done in [13]. In a subsequent paper [15], we will investigate the influence of $N N$ - and $\pi N$ rescattering on the various asymmetries of the semi-exclusive differential cross section of incoherent pion photoproduction on the deuteron. 


\section{Acknowledgments}

We would like to thank Michael Schwamb for interesting discussions and a careful reading of the manuscript. This work was supported by the Deutsche Forschungsgemeinschaft (SFB 443).

\section{APPENDIX: EXPLICIT EXPRESSIONS FOR THE VARIOUS POLARIZATION ASYMMETRIES}

We list here the explicit hermitean, bilinear forms in terms of the $t$-matrix elements for cross section and the various asymmetries:

1. The semi-exclusive differential cross section

$$
\frac{d^{3} \sigma_{0}}{d q d \Omega_{q}}=\frac{1}{3} \int d \Omega_{p} c\left(\omega, q, \Omega_{q}, \Omega_{p}\right) \sum_{s m_{s} m_{d}}\left|t_{s m_{s} 1 m_{d}}\right|^{2} .
$$

2. The photon asymmetry for linearly polarized photons and unpolarized deuterons

$$
\widetilde{\Sigma}^{l} \frac{d^{3} \sigma_{0}}{d q d \Omega_{q}}=-\frac{1}{3} \int d \Omega_{p} c\left(\omega, q, \Omega_{q}, \Omega_{p}\right) \sum_{s m_{s} m_{d}} t_{s m_{s} 1 m_{d}}^{*} t_{s m_{s}-1 m_{d}}
$$

3. The target asymmetry for vector polarized deuterons and unpolarized photons

$$
\widetilde{T}_{11}^{0} \frac{d^{3} \sigma_{0}}{d q d \Omega_{q}}=\sqrt{\frac{2}{3}} \int d \Omega_{p} c\left(\omega, q, \Omega_{q}, \Omega_{p}\right) \Im m \sum_{s m_{s}}\left(t_{s m_{s} 1-1}^{*} t_{s m_{s} 10}+t_{s m_{s} 10}^{*} t_{s m_{s} 11}\right) .
$$

4. The target asymmetries for tensor polarized deuterons and unpolarized photons

$$
\begin{aligned}
& \widetilde{T}_{20}^{0} \frac{d^{3} \sigma_{0}}{d q d \Omega_{q}}=\frac{1}{3 \sqrt{2}} \int d \Omega_{p} c\left(\omega, q, \Omega_{q}, \Omega_{p}\right) \sum_{s m_{s}}\left(\left|t_{s m_{s} 11}\right|^{2}+\left|t_{s m_{s} 1-1}\right|^{2}-2\left|t_{s m_{s} 10}\right|^{2}\right), \\
& \widetilde{T}_{21}^{0} \frac{d^{3} \sigma_{0}}{d q d \Omega_{q}}=\sqrt{\frac{2}{3}} \int d \Omega_{p} c\left(\omega, q, \Omega_{q}, \Omega_{p}\right) \Re e \sum_{s m_{s}}\left(t_{s m_{s} 1-1}^{*} t_{s m_{s} 10}-t_{s m_{s} 10}^{*} t_{s m_{s} 11}\right), \\
& \widetilde{T}_{22}^{0} \frac{d^{3} \sigma_{0}}{d q d \Omega_{q}}=\frac{2}{\sqrt{3}} \int d \Omega_{p} c\left(\omega, q, \Omega_{q}, \Omega_{p}\right) \Re e \sum_{s m_{s}} t_{s m_{s} 1-1}^{*} t_{s m_{s} 11} .
\end{aligned}
$$

5. The beam-target asymmetries for circularly polarized photons and vector polarized deuterons

$$
\begin{aligned}
& \widetilde{T}_{10}^{c} \frac{d^{3} \sigma_{0}}{d q d \Omega_{q}}=\frac{1}{\sqrt{6}} \int d \Omega_{p} c\left(\omega, q, \Omega_{q}, \Omega_{p}\right) \sum_{s m_{s}}\left(\left|t_{s m_{s} 11}\right|^{2}-\left|t_{s m_{s} 1-1}\right|^{2}\right), \\
& \widetilde{T}_{11}^{c} \frac{d^{3} \sigma_{0}}{d q d \Omega_{q}}=-\sqrt{\frac{2}{3}} \int d \Omega_{p} c\left(\omega, q, \Omega_{q}, \Omega_{p}\right) \Re e \sum_{s m_{s}}\left(t_{s m_{s} 1-1}^{*} t_{s m_{s} 10}+t_{s m_{s} 10}^{*} t_{s m_{s} 11}\right) .
\end{aligned}
$$

6. The beam-target asymmetries for circularly polarized photons and tensor polarized deuterons

$$
\begin{aligned}
& \widetilde{T}_{21}^{c} \frac{d^{3} \sigma_{0}}{d q d \Omega_{q}}=\sqrt{\frac{2}{3}} \int d \Omega_{p} c\left(\omega, q, \Omega_{q}, \Omega_{p}\right) \Im m \sum_{s m_{s}}\left(t_{s m_{s} 10}^{*} t_{s m_{s} 11}-t_{s m_{s} 1-1}^{*} t_{s m_{s} 10}\right), \\
& \widetilde{T}_{22}^{c} \frac{d^{3} \sigma_{0}}{d q d \Omega_{q}}=-\frac{2}{\sqrt{3}} \int d \Omega_{p} c\left(\omega, q, \Omega_{q}, \Omega_{p}\right) \Im m \sum_{s m_{s}} t_{s m_{s} 1-1}^{*} t_{s m_{s} 11} .
\end{aligned}
$$


7. The beam-target asymmetries for linearly polarized photons and vector polarized deuterons

$$
\begin{aligned}
\widetilde{T}_{10}^{l} \frac{d^{3} \sigma_{0}}{d q d \Omega_{q}} & =\sqrt{\frac{2}{3}} \int d \Omega_{p} c\left(\omega, q, \Omega_{q}, \Omega_{p}\right) \Im m \sum_{s m_{s}}\left(t_{s m_{s} 11}^{*} t_{s m_{s}-11}\right), \\
\widetilde{T}_{11}^{l} \frac{d^{3} \sigma_{0}}{d q d \Omega_{q}} & =-\sqrt{\frac{2}{3}} \int d \Omega_{p} c\left(\omega, q, \Omega_{q}, \Omega_{p}\right) \Im m \sum_{s m_{s}}\left(t_{s m_{s} 1-1}^{*} t_{s m_{s}-10}\right), \\
\widetilde{T}_{1-1}^{l} \frac{d^{3} \sigma_{0}}{d q d \Omega_{q}} & =\sqrt{\frac{2}{3}} \int d \Omega_{p} c\left(\omega, q, \Omega_{q}, \Omega_{p}\right) \Im m \sum_{s m_{s}}\left(t_{s m_{s} 11}^{*} t_{s m_{s}-10}\right) .
\end{aligned}
$$

8. The beam-target asymmetries for linearly polarized photons and tensor polarized deuterons

$$
\begin{aligned}
\widetilde{T}_{20}^{l} \frac{d^{3} \sigma_{0}}{d q d \Omega_{q}} & =\frac{\sqrt{2}}{3} \int d \Omega_{p} c\left(\omega, q, \Omega_{q}, \Omega_{p}\right) \Re e \sum_{s m_{s}}\left(t_{s m_{s} 10}^{*} t_{s m_{s}-10}-t_{s m_{s} 11}^{*} t_{s m_{s}-11}\right), \\
\widetilde{T}_{21}^{l} \frac{d^{3} \sigma_{0}}{d q d \Omega_{q}} & =\sqrt{\frac{2}{3}} \int d \Omega_{p} c\left(\omega, q, \Omega_{q}, \Omega_{p}\right) \Re e \sum_{s m_{s}}\left(t_{s m_{s} 10}^{*} t_{s m_{s}-11}\right), \\
\widetilde{T}_{2-1}^{l} \frac{d^{3} \sigma_{0}}{d q d \Omega_{q}} & =\sqrt{\frac{2}{3}} \int d \Omega_{p} c\left(\omega, q, \Omega_{q}, \Omega_{p}\right) \Re e \sum_{s m_{s}}\left(t_{s m_{s} 10}^{*} t_{s m_{s}-1-1}\right) \\
\widetilde{T}_{22}^{l} \frac{d^{3} \sigma_{0}}{d q d \Omega_{q}} & =-\frac{1}{\sqrt{3}} \int d \Omega_{p} c\left(\omega, q, \Omega_{q}, \Omega_{p}\right) \sum_{s m_{s}} t_{s m_{s} 1-1}^{*} t_{s m_{s}-11} \\
\widetilde{T}_{2-2}^{l} \frac{d^{3} \sigma_{0}}{d q d \Omega_{q}} & =-\frac{1}{\sqrt{3}} \int d \Omega_{p} c\left(\omega, q, \Omega_{q}, \Omega_{p}\right) \sum_{s m_{s}} t_{s m_{s} 11}^{*} t_{s m_{s}-1-1} .
\end{aligned}
$$

[1] E.M. Darwish, H. Arenhövel, and M. Schwamb, Eur. Phys. J. A 16, 111 (2003).

[2] A. Sandorfi for the LEGS-Collaboration, private communication.

[3] P. Pedroni for the A2-Collaboration, private communication.

[4] M.I. Levchuk, V.A. Petrun'kin, and M. Schumacher, Z. Phys. A 355, 317 (1996).

[5] E.M. Darwish, H. Arenhövel, and M. Schwamb, Eur. Phys. J. A 17, 513 (2003).

[6] H. Arenhövel, A. Fix, and M. Schwamb, Phys. Rev. Lett. 93, 202301 (2004).

[7] A. Loginov, A.Sidorov, and V. Stibunov, Phys. Atom. Nucl. 63,391 (2000) (Yad. Fiz. 63, 459 (2000)).

[8] E.M. Darwish, J. Phys. G 31, 105 (2004).

[9] E.M. Darwish, Nucl. Phys. A 735, 200 (2005).

[10] E.M. Darwish, Nucl. Phys. A 748, 596 (2005).

[11] E.M. Darwish, nucl-th/0504031

[12] E.M. Darwish and A. Salam, nucl-th/0505002

[13] H. Arenhövel, Few-Body Syst. 4, 55 (1988).

[14] F. Blaazer, B.L.G. Bakker, and H.J. Boersma, Nucl. Phys. A 568, 681 (1994).

[15] A. Fix and H. Arenhövel, in preparation.

[16] E.M. Rose, Elementary Theory of Angular Momentum, Wiley New York 1957.

[17] B.A. Robson, The Theory of Polarization Phenomena, Clarendon Press, Oxford 1974. 